\title{
SUMS OF ZEROS OF SOLUTIONS TO SECOND ORDER DIFFERENTIAL EQUATIONS WITH POLYNOMIAL COEFFICIENTS
}

\author{
Michael GiL' \\ Ben Gurion University of the Negev, Israel
}

\begin{abstract}
We consider the equation $u^{\prime \prime}=P(z) u$, where $P(z)$ is a polynomial. Let $z_{k}(u), k=1,2, \ldots$ be the zeros of a solution $u(z)$ to that equation. Inequalities for the sums $\sum_{k=1}^{j} \frac{1}{\left|z_{k}(u)\right|}(j=1,2, \ldots)$ are derived. They considerably improve the previous result of the author. Some applications of the obtained bounds are also discussed. An illustrative example is presented. It shows that the suggested results are sharp.
\end{abstract}

\section{Introduction AND STATEMENT OF THE MAIN RESUlT}

In the present paper we consider linear differential equations with polynomial coefficients in the complex domain. The literature devoted to the zeros of solutions of such equations is very rich. Here the main tool is the Nevanlinna theory. An excellent exposition of the Nevanlinna theory and its applications to differential equations is given in the book [22]. In that book, in particular, the well-known results of Bank ([3]), Brüggemann ([5]), Hellerstein ([18]) and other mathematicians are featured. The classical comparison principle for zeros of ODE in the complex plane is presented in [20]. The real zeros of solutions to equations with polynomial coefficients were investigated in the very interesting papers by Gundersen ([17]), Eremenko and Merenkov ([9]), and by C. Z. Huang ([21]). In connection with recent results see also the papers $[4,10,11],[23]-[27]$. In particular, in the paper [26], the authors study the convergence of the zeros of a non-trivial (entire) solution to the linear

2010 Mathematics Subject Classification. 34C10, 34A30.

Key words and phrases. Linear differential equation in the complex plane, bounds for zeros of solutions. 
differential equation

$$
f^{\prime \prime}+\left\{Q_{1}(z) e^{P_{1}(z)}+Q_{2}(z) e^{P_{2}(z)}+Q_{3}(z) e^{P_{3}(z)}\right\} f=0
$$

where $P_{j}$ are polynomials of degree $n \geq 1$ and $Q_{j}(\not \equiv 0)$ are entire functions of order less than $n(j=1,2,3)$. In the paper [11], by certain separation and comparison results, estimates for the counting functions of the zeros of solutions to $n$ th-order linear differential equations are deduced. It is proved that these estimates are in any possible case the sharpest ones, and they generalize known results for the zeros of solutions to third- and fourth-order linear differential equations. The remarkable results on the zeros of a wide class of ordinary differential equations with polynomial coefficients whose solutions are classical orthogonal polynomials were established by N. Anghel ([1]). Besides, he had derived results connected with the equations of mathematical physics. In addition, N. Anghel ([2]), investigated the following question: when is an entire function of finite order, solution to a complex 2nd order homogeneous linear differential equation with polynomial coefficients? He gives two (equivalent) answers to this question, one of which involves certain Stieltjes-like relations for the zeros of solutions, the second one requires the vanishing of all but finitely many suitable expressions constructed via the relations of the sums of the zeros of the function derived in [13].

Certainly, we could not survey the whole subject here and refer the reader to the above listed publications and references given therein. In the above cited works mainly the asymptotic distributions of zeros and counting functions of zeros are investigated. At the same time, bounds for the zeros of solutions are very important in various applications. But to the best of our knowledge, they have been investigated considerably less than the asymptotic distributions. In the paper [14] the author has established bounds for the sums of the zeros of solutions for the second order equations with polynomial coefficients. In the interesting paper [6], some results from [14] have been extended to the equation $u^{(m)}=P(z) u$, where $P$ is a polynomial and $m>2$. In [16] the main result from [14] is extended to the second order ODE with non-polynomial coefficients. Perturbations of the zeros of solutions to second order differential equations with polynomial coefficients were investigated in the paper [16].

In the present paper, we considerably refine the main result from [14]. Note that, the proof of the main result of the present paper-Theorem 1.1 is considerably different from the proof of the one from [14]. In addition, we estimate the zero free domains. That estimation supplements the well-known results from [20] as well as the results from the paper of Eloe and Henderson [8] on the positivity of solutions for higher order ordinary differential equations, since the positivity of solutions implies the absence of zeros.

Consider the equation

$$
u^{\prime \prime}=P(z) u, u(0)=u_{0}, u^{\prime}(0)=u_{1} \quad\left(u_{0}, u_{1} \in \mathbb{C} ; u_{0} \neq 0\right),
$$


where

$$
P(z)=\sum_{k=0}^{n} c_{k} z^{k} \quad\left(c_{n} \neq 0\right)
$$

is a polynomial with complex coefficients. As it is well-known [22], the zeros of solutions to (1.1) are simple. Enumerate the zeros $z_{k}(u)$ of $u$ in order of increasing modulus: $\left|z_{k}(u)\right| \leq\left|z_{k+1}(u)\right|(k=1,2, \ldots)$. Put

$$
s(P)=\left(\sum_{k=0}^{n}\left|c_{k}\right|\right)^{1 / 2}, \beta(P)=((1+n / 2) s(P) e)^{2 /(n+2)}
$$

and

$$
C_{0}(P):=\beta(P) e^{s(P)}+\frac{\left|u_{1}\right|}{\left|u_{0}\right|}\left(1+4 e^{s(P)}\right)
$$

THEOREM 1.1. If $u_{0} \neq 0$, then

$$
\sum_{k=1}^{j} \frac{1}{\left|z_{k}(u)\right|} \leq C_{0}(P)+\sqrt{2} \beta(P) \sum_{k=1}^{j} \frac{1}{(k+1)^{\frac{2}{n+2}}}(j=1,2, \ldots) .
$$

The proof of this theorem is presented in the next two sections.

To estimate the sharpness of the theorem, consider the equation

$$
u^{\prime \prime}+u=0, u(0)=1, u^{\prime}(0)=0 \text {. }
$$

Then $u(z)=\cos (z)$ and its zeros are $\pi(k+1 / 2)(k=0, \pm 1, \pm 2, \ldots)$. So

$$
\sum_{k=1}^{2 j+1} \frac{1}{\left|z_{k}(u)\right|}=\frac{1}{\pi}\left(\frac{1}{j+1 / 2}+2 \sum_{k=0}^{j-1} \frac{1}{k+1 / 2}\right) .
$$

In the considered case $n=0, s(P)=1, \beta(P)=e, C_{0}(P)=e^{2}$. Therefore, Theorem 1.1 gives us the inequality

$$
\sum_{k=1}^{2 j+1} \frac{1}{\left|z_{k}(u)\right|} \leq e^{2}+\sqrt{2} e \sum_{k=1}^{2 j+1} \frac{1}{k+1}(j=1,2, \ldots) .
$$

We can see that (1.2) and (1.3) are asymptotically equivalent.

\section{Estimates For solutions}

Consider the equation

$$
\frac{d^{2} u}{d z^{2}}=Q(z) u, \quad u(0)=u_{0}, u^{\prime}(0)=u_{1} \quad\left(u_{0}, u_{1} \in \mathbb{C}\right),
$$

where

$$
Q(z)=\sum_{k=0}^{\infty} c_{k} z^{k}\left(c_{0} \neq 0\right)
$$

is an entire function. Put $M_{f}(r)=\sup _{|z| \leq r}|f(z)|$ for a function $f(z)$. 
LEMma 2.1. A solution $u(z)$ of equation (2.1) satisfies the inequality $M_{u}(r) \leq\left(\left|u_{0}\right|+r\left|u_{1}\right|\right) \cosh (r \sqrt{q(r)}) \quad\left(r \geq 0 ; \cosh (r)=\frac{1}{2}\left(e^{r}+e^{-r}\right)\right)$, where

$$
q(r)=\sum_{k=0}^{\infty}\left|c_{k}\right| r^{k}
$$

Proof. For a fixed $t \in[0,2 \pi)$ and $z=r e^{i t}$ we have

$$
e^{-2 i t} \frac{d^{2} u\left(r e^{i t}\right)}{d r^{2}}=Q\left(r e^{i t}\right) u\left(r e^{i t}\right) .
$$

Integrating twice this equation in $r$, we obtain

$$
e^{-2 i t} u\left(r e^{i t}\right)=e^{-2 i t}\left(u_{0}+r u_{1}\right)+\int_{0}^{r}(r-s) Q\left(s e^{i t}\right) u\left(s e^{i t}\right) d s .
$$

Hence,

$$
\left|u\left(r e^{i t}\right)\right| \leq\left|u_{0}\right|+r\left|u_{1}\right|+\int_{0}^{r}(r-s) q(s)\left|u\left(s e^{i t}\right)\right| d s .
$$

Due to the comparison lemma [7, Lemma III.2.1], we have $\left|u\left(r e^{i t}\right)\right| \leq v(r)$, where $v(r)$ is a solution of the equation

$$
v(r)=w(r)+\int_{0}^{r}(r-s) q(s) v(s) d s=w(r)+V v(r)
$$

where $w(r)=\left|u_{0}\right|+r\left|u_{1}\right|$ and $V$ is the Volerra operator defined by

$$
(V v)(r)=\int_{0}^{r}(r-s) q(s) v(s) d s .
$$

So

$$
v=\sum_{k=0}^{\infty} V^{k} w
$$

But for any positive nondecreasing $h(r)$ we have

$$
V h(r)=\int_{0}^{r}(r-s) q(s) h(s) d s \leq h(r) q(r) \int_{0}^{r}(r-s) d s .
$$

Hence,

$$
\begin{aligned}
V^{m} h(r) & \leq q^{m}(r) h(r) \int_{0}^{r} \int_{0}^{r_{1}} \ldots \int_{0}^{r_{m-1}}\left(r-r_{1}\right) \ldots\left(r_{m-1}-r_{m}\right) d r_{1} \ldots d r_{m} \\
& =q^{m}(r) h(r) \frac{r^{2 m}}{(2 m) !} .
\end{aligned}
$$

Thus from (2.4) it follows

$$
v(r) \leq\left(\left|u_{0}\right|+r\left|u_{1}\right|\right) \sum_{k=0}^{\infty} \frac{q^{k}(r) r^{2 k}}{(2 k) !} .
$$


But

$$
\sum_{k=0}^{\infty} \frac{q^{k}(r) r^{2 k}}{(2 k) !}=\cosh (r \sqrt{q(r)}) .
$$

This implies the required result.

It should be noted that the inequality stated in Lemma 2.1 can be proved by Herold's comparison theorem [19].

Consider again the equation

$$
\frac{d^{2} u}{d z^{2}}=P(z) u, \quad u(0)=u_{0}, u^{\prime}(0)=u_{1} \quad\left(u_{0}, u_{1} \in \mathbb{C}\right) .
$$

In this case

$$
q(r)=\hat{p}(r):=\sum_{k=0}^{n}\left|c_{k}\right| r^{k}
$$

In addition

$$
r \sqrt{\hat{p}(r)} \leq \sqrt{\hat{p}(1)}\left(1+r^{n / 2+1}\right) \quad(r>0)
$$

and

$$
\cosh (r \sqrt{\hat{p}(r)}) \leq \exp \left[\sqrt{\hat{p}(1)}\left(1+r^{n / 2+1}\right)\right] .
$$

Sine $\hat{p}(1)=s^{2}(P)$, Lemma 2.1 yields

Corollary 2.2. A solution of equation (2.5) satisfies the inequality

$$
M_{u}(r) \leq\left(\left|u_{0}\right|+r\left|u_{1}\right|\right) e^{s(P)\left(1+r^{n / 2+1}\right)} .
$$

The previous corollary is sharp: as it is well-known a solution of equation $(2.5)$ is an entire function of order no more than $(n+2) / 2$, see, e.g. [22, Proposition 5.1]. Besides, our proof is absolutely different.

\section{Proof of Theorem 1.1}

LEMMA 3.1. Let an entire function

$$
f(z)=\sum_{k=0}^{\infty} f_{k} z^{k}
$$

satisfy the inequality

$$
M_{f}(r) \leq\left(D_{1}+D_{2} r\right) \exp \left[B r^{\rho}\right]
$$

$$
\left(D_{1}, D_{2}=\text { const } \geq 0 ; B=\text { const }>0 ; \rho \geq 1 ; r>0\right) .
$$

Then its Taylor coefficients are subject to the inequalities

$$
\left|f_{j}\right| \leq D_{1} \frac{(e B \rho)^{j / \rho}}{(j !)^{1 / \rho}}+D_{2} \frac{(e B \rho)^{(j-1) / \rho}}{[(j-1) !]^{1 / \rho}}(j \geq 2) .
$$


Proof. By the well-known inequality for the coefficients of a power series

$$
\left|f_{j}\right| \leq \frac{M_{f}(r)}{r^{j}} \leq\left(D_{1}+D_{2} r\right) \frac{e^{B r^{\rho}}}{r^{j}} .
$$

Employing the usual method for finding extrema it is easy to see that the function $r^{-j} e^{B r^{\rho}}(j \geq 1)$ takes its smallest value in the range $r>0$ for $r_{j}=\left(\frac{j}{B \rho}\right)^{1 / \rho}$ and therefore

$$
\left|f_{j}\right| \leq \frac{M_{f}\left(r_{j}\right)}{r_{j}^{j}} \leq D_{1}\left(\frac{e B \rho}{j}\right)^{j / \rho}+D_{2}\left(\frac{e B \rho}{j-1}\right)^{(j-1) / \rho} \quad(j \geq 2) .
$$

Hence, due to the well known inequality, $j^{j} \geq j$ ! $(j \geq 1)$, we have $(3.2)$, as claimed.

Note that Lemma 3.1 can be proved also by the classical Valiron-Wiman theory, cf. [25, page 11,Q. 67 (1)].

Now let us consider the entire function

$$
f(z)=\sum_{k=0}^{\infty} \frac{d_{k} z^{k}}{(k !)^{1 / \rho}}\left(\rho \geq 1, \lambda \in \mathbb{C}, d_{0}=1, d_{k} \in \mathbb{C}\right) .
$$

Assume that

$$
\theta(f):=\left[\sum_{k=1}^{\infty}\left|d_{k}\right|^{2}\right]^{1 / 2}<\infty .
$$

Theorem 3.2. Let $f$ be defined by (3.3) and condition (3.4) hold. Then

$$
\sum_{k=1}^{j} \frac{1}{\left|z_{k}(f)\right|} \leq \theta(f)+\sum_{k=1}^{j} \frac{1}{(k+1)^{1 / \rho}} \quad(j=1,2, \ldots) .
$$

This theorem is proved in [12] (see also [13, Section 5.1]).

Denote

$$
b:=(e B \rho)^{1 / \rho} \text { and } \tau_{\rho}:=\left(\sum_{j=2}^{\infty} \frac{j^{2 / \rho}}{2^{j}}\right)^{1 / 2} .
$$

LEMMA 3.3. Let an entire function $f$ satisfy the conditions (3.1) and $f(0)=1$. Then

$$
\sum_{k=1}^{j} \frac{1}{\left|z_{k}(f)\right|} \leq\left|f_{1}\right|+D_{1} b+\sqrt{2} D_{2} \tau_{\rho}+\sqrt{2} b \sum_{k=1}^{j} \frac{1}{(k+1)^{1 / \rho}} \quad(j=1,2, \ldots) .
$$

Proof. Due to Lemma 3.1

$$
\left|f_{k}\right| \leq \frac{D_{1} b^{k}}{(k !)^{1 / \rho}}+\frac{D_{2} b^{k-1}}{[(k-1) !]^{1 / \rho}}=\frac{1}{(k !)^{1 / \rho}}\left(D_{1} b^{k}+D_{2} b^{k-1} k^{1 / \rho}\right) \quad(k \geq 2) \text {. }
$$


Consider the function

$$
f_{\chi}(z)=f(\chi z)=1+\sum_{k=1}^{\infty} \frac{f_{k} \chi^{k} z^{k}}{(k !)^{1 / \rho}}
$$

with $\chi=\frac{1}{b \sqrt{2}}$. We have

$$
\begin{aligned}
\theta^{2}\left(f_{\chi}\right) & =\sum_{j=1}^{\infty}\left|\chi^{j} f_{j}\right|^{2} \leq\left|f_{1}\right|^{2} \chi^{2}+\sum_{j=2}^{\infty} \frac{1}{(b \sqrt{2})^{2 j}}\left(D_{1} b^{j}+D_{2} b^{j-1} j^{1 / \rho}\right)^{2} \\
& =\left|f_{1}\right|^{2} \chi^{2}+\sum_{j=2}^{\infty} \frac{1}{2^{j}}\left(D_{1}+\frac{D_{2}}{b} j^{1 / \rho}\right)^{2}
\end{aligned}
$$

Hence, by the triangle inequality

$$
\theta\left(f_{\chi}\right) \leq\left|f_{1}\right| \chi+D_{1}\left(\sum_{j=2}^{\infty} \frac{1}{2^{j}}\right)^{1 / 2}+\frac{D_{2} \tau_{\rho}}{b}=\left|f_{1}\right| \chi+\frac{D_{1}}{\sqrt{2}}+\frac{D_{2} \tau_{\rho}}{b} .
$$

Thus due to Theorem 3.2 we obtain

$$
\sum_{k=1}^{j} \frac{1}{\left|z_{k}\left(f_{\chi}\right)\right|} \leq\left|f_{1}\right| \chi+\frac{D_{1}}{\sqrt{2}}+D_{2} \frac{\tau_{\rho}}{b}+\sum_{k=1}^{j} \frac{1}{(k+1)^{1 / \rho}}(j=1,2, \ldots) .
$$

Since $\chi z_{k}\left(f_{\chi}\right)=z_{k}(f)$, the lemma is proved.

Proof of Theorem 1.1. Delete $(1.1)$ by $u(0)$. According to Corollary 2.2 we take $B=s(P), \rho=1+n / 2$,

$$
D_{1}=\frac{1}{\left|u_{0}\right|} e^{s(P)} \text { and } D_{2}=\frac{\left|u_{1}\right|}{\left|u_{0}\right|} e^{s(P)} .
$$

Then $b=\beta(P)$. In addition,

$$
\tau_{1+n / 2}^{2}=\sum_{j=2}^{\infty} \frac{j^{4 /(n+2)}}{2^{j}} \leq \frac{1}{2} \sum_{j=2}^{\infty} \frac{j(j-1)}{2^{j-2}}=8(n \geq 0) .
$$

Now Lemma 3.3 implies

$$
\begin{aligned}
\sum_{k=1}^{j} \frac{1}{\left|z_{k}(u)\right|} & \leq \beta(P) e^{s(P)}+\frac{\left|u_{1}\right|}{\left|u_{0}\right|}\left(1+4 e^{s(P)}\right)+\sqrt{2} \beta(P) \sum_{k=1}^{j} \frac{1}{(k+1)^{2 /(n+2)}} \\
& =C_{0}(P)+\sqrt{2} \beta(P) \sum_{k=1}^{j} \frac{1}{(k+1)^{2 /(2+n)}} \quad(j=1,2, \ldots),
\end{aligned}
$$

as claimed. 


\section{Applichtions of Theorem 1.1}

Again $u(z)$ is a solution of equation (1.1). Everywhere in this section we assume that $u(0) \neq 0$ and for the brevity let

$$
\hat{\beta}=\sqrt{2} \beta(P) \text { and } \gamma=\frac{2}{n+2} .
$$

Since $\left|z_{k}(u)\right| \leq\left|z_{k+1}(u)\right|$, Theorem 1.1 implies that

$$
j\left|z_{j}(u)\right|^{-1} \leq C_{0}(P)+\hat{\beta} \sum_{k=1}^{j} \frac{1}{(k+1)^{\gamma}} \quad(j=1,2, \ldots) .
$$

But

$$
\sum_{k=1}^{j}(k+1)^{-\gamma} \leq \int_{1}^{j+1} \frac{d x}{x^{\gamma}}=\frac{(1+j)^{1-\gamma}-1}{1-\gamma} \quad(0<\gamma<1) .
$$

Denote by $\nu(f, a)(a>0)$ the counting function of the zeros of $f$ in the circle $|z| \leq a$. We thus get

Corollary 4.1. Let $u(0) \neq 0$ and $n>0$. Then with the notation

$$
\eta_{j}(u):=\frac{j}{C_{0}(P)+\hat{\beta} \frac{(1+j)^{1-\gamma}-1}{1-\gamma}},
$$

the inequality $\left|z_{j}(u)\right| \geq \eta_{j}(u)$ holds and thus $\nu(u, a) \leq j$ for any positive $a \leq \eta_{j}(u)(j=1,2, \ldots)$.

Furthermore, put

$$
\vartheta_{1}=C_{0}(P)+\frac{\hat{\beta}}{2^{\gamma}} \text { and } \vartheta_{k}=\frac{\hat{\beta}}{(k+1)^{\gamma}}(k=2,3, \ldots) .
$$

Theorem 1.1 and Lemma 1.2.1 from [13] yield

Corollary 4.2. Let $\phi(t)(0 \leq t<\infty)$ be a continuous convex scalarvalued function, such that $\phi(0)=0$. Then

$$
\sum_{k=1}^{j} \phi\left(\left|z_{k}(u)\right|^{-1}\right) \leq \sum_{k=1}^{j} \phi\left(\vartheta_{k}\right) \quad(j=1,2, \ldots) .
$$

In particular, for any $p \geq 1$ and $j=2,3, \ldots$, we have

$$
\sum_{k=1}^{j} \frac{1}{\left|z_{k}(u)\right|^{p}} \leq \sum_{k=1}^{j} \vartheta_{k}^{p}
$$

and therefore

$$
\sum_{k=1}^{\infty} \frac{1}{\left|z_{k}(u)\right|^{p}}<\infty
$$

provided that $p>n+1 / 2$. 
In addition, making use Theorem 1.1 and [13, Lemma 1.2.2], we obtain our next result.

Corollary 4.3. Let $\Phi\left(t_{1}, t_{2}, \ldots, t_{j}\right)$ be a function defined on domain Introduce a scalar-valued function $\Phi\left(t_{1}, t_{2}, \ldots, t_{j}\right)$ with an integer $j$ defined on the domain

and satisfying the condition

$$
-\infty<t_{j} \leq t_{j-1} \ldots \leq t_{2} \leq t_{1}<\infty
$$

$$
\frac{\partial \Phi}{\partial t_{1}}>\frac{\partial \Phi}{\partial t_{2}}>\ldots>\frac{\partial \Phi}{\partial t_{j}}>0 \text { for } t_{1}>t_{2}>\ldots>t_{j}>-\infty
$$

Then

$$
\Phi\left(\frac{1}{\left|z_{1}(u)\right|}, \ldots, \frac{1}{\left|z_{j}(u)\right|}\right) \leq \Phi\left(\vartheta_{1}, \ldots, \vartheta_{j}\right) .
$$

In particular, let $\left\{d_{k}\right\}_{k=1}^{\infty}$ be a decreasing sequence of positive numbers with $d_{1}=1$. Then the previous corollary and Theorem 1.1 yield the inequality

$$
\sum_{k=1}^{j} \frac{d_{k}}{\left|z_{k}(u)\right|} \leq C_{0}(P)+\hat{\beta} \sum_{k=1}^{j} \frac{d_{k}}{(k+1)^{\gamma}}(j=1,2, \ldots) .
$$

Finally we improve Corollary 4.1 in the case $j=1$. Besides, $n \geq 0$. Due to [13, Theorem 5.12.1], we can write

$$
\inf _{j}\left|z_{j}(u)\right| \geq 1 /\left(1+\max _{j}\left|a_{j}\right|\right),
$$

where $a_{j}(j=1,2, \ldots)$ are the Taylor coefficients at zero of $v(z)=u(z) / u(0)$. We have $\left|a_{j}\right| \leq M_{v}(1)$. Now Corollary 2.2 implies

$$
\inf _{j}\left|z_{j}(u)\right| \geq \frac{1}{\left(1+\frac{\left|u_{1}\right|}{\left|u_{0}\right|}\right) e^{2 s(P)}} .
$$

The just obtained result gives us a bound for the zero-free domain.

\section{REFERENCES}

[1] N. Anghel, Stieltjes-Calogero-Gil' relations associated to entire functions of finite order, J. Math. Phys. 51 (2010), 053509, 9 pp.

[2] N. Anghel, Entire functions of finite order as solutions to certain complex linear differential equations, Proc. Amer. Math. Soc. 140 (2012), 2319-2332.

[3] S. Bank, A note on the location of complex zeros of solutions of linear differential equations, Complex Variables Theory Appl. 12 (1989), 159-167.

[4] B. Belaïdi and A. E. Farissi, Differential polynomials generated by some complex linear differential equations with meromorphic coefficients, Glas. Mat. Ser. III 43(63) (2008), 363-373.

[5] F. Brüggemann, On the zeros of fundamental systems of linear differential equations with polynomial coefficients, Complex Variables Theory Appl. 15 (1990), 159-166.

[6] T.-B. Cao, K. Liu and H.-Y. Xu, Bounds for the sums of zeros of solutions of $u(m)=P(z) u$ where $P$ is a polynomial, Electron. J. Qual. Theory Differ. Equ. 2011 (2011), no. 60, 10 pp. 
[7] Yu L. Daleckii and M. G. Krein, Stability of solutions of differential equations in Banach space, Amer. Math. Soc., Providence, R. I. 1974.

[8] P. W. Eloe and J. Henderson, Positive solutions for higher order ordinary differential equations, Electron. J. Differential Equations 1995 (1995), no. 03, 8 pp.

[9] A. Eremenko and S. Merenkov. Nevanlinna functions with real zeros, Illinois J. Math. 49 (2005), 1093-1110 (electronic).

[10] A. E. Farissi and B. Belaïdi, On oscillation theorems for differential polynomials, Electron. J. Qual. Theory Differ. Equ. 22 (2009), No. 22, 10 pp.

[11] M. Gaudenzi, On the number of the zeros of solutions of a linear differential equation, J. Math. Anal. Appl. 221 (1998), 306-325.

[12] M. I. Gil', Inequalities for zeros of entire functions, J. Inequal. Appl. 6 (2001), 463471.

[13] M. I. Gil', Localization and perturbation of zeros of entire functions, CRC Press, Boca Raton, 2010.

[14] M. I. Gil', Bounds for zeros of solutions of second order differential equations with polynomial coefficients, Results Math. 59 (2011), 115-124.

[15] M. I. Gil', Perturbation of zeros of solutions to second order differential equations with polynomial coefficients, Acta Math. Sci. Ser. B Engl. Ed. 32 (2012), 1083-1092.

[16] M. I. Gil', Sums of zeros of solutions to second order ODE with non-polynomial coefficients, Electron. J. Differential Equations 2012 (2012), no. 107, 8 pp.

[17] G. G. Gundersen, On the real zeros of solutions of $f$ " $+A(z) f=0$ where $A(z)$ is entire, Ann. Acad. Sci. Fenn. Ser. A I Math. 11 (1986), 275-294

[18] S. Hellerstein and J. Rossi, On the distribution of zeros of solutions of second-order differential equations, Complex Variables Theory Appl. 13 (1989), 99-109.

[19] H. Herold, Ein Vergleichssatz für komplexe lineare Differentialgleichungen, Math. Z. 126 (1972), 91-94.

[20] E. Hille, Lectures on ordinary differential equations, Addison Wesley Publ. Co., Ontario, 1969.

[21] C. Z. Huang, Real zeros of solutions of second order linear differential equations, Kodai Math. J. 14 (1991), 113-122.

[22] I. Laine, Nevanlinna theory and complex differential equations, Walter de Gruyter Berlin, 1993.

[23] C.-H. Lin, Y. Sibuya and T. Tabara, Zeros of solutions of a second order linear differential equation with polynomial coefficients, Funkcial. Ekvac. 36 (1993), 375384.

[24] G. M. Muminov, On the zeros of solutions of the differential equation $\omega^{(2 m)}+p(z) \omega=$ 0, Demonstratio Math. 35 (2002), 41-48.

[25] G. Polya and G. Szegö, Problems and theorems in analysis. Vol. II, Springer-Verlag, New York, 1976.

[26] J. Tu and Z. X. Chen, Zeros of solutions of certain second order linear differential equation, J. Math. Anal. Appl. 332 (2007), 279-291.

[27] J. F. Xu and H. X. Yi, Solutions of higher order linear differential equations in an angle, Appl. Math. Lett. 22 (2009), 484-489.

M. Gil'

Department of Mathematics

Ben Gurion University of the Negev

P.0. Box 653, Beer-Sheva 84105

Israel

E-mail: gilmi@bezeqint.net

Received: 3.1.2014. 